\section{Materials for Optical Data Storage}

\section{Hans Coufal and Lisa Dhar, Guest Editors}

\begin{abstract}
The development of recording materials has been a critical component in the advancement of optical data storage. The continual need for improved performance in both consumer and business applications has pushed forward the capabilities of optical storage. In this issue of MRS Bulletin, we review some of the important developments in the materials behind established technologies such as compact discs, digital versatile discs, and magneto-optical recording; the emerging technology of blue recording; and two technologies that seek to extend the performance roadmap for optical storage, multilayer and holographic recording.
\end{abstract}

Keywords: magneto-optic, memory, optical.
Optical storage has become ubiquitous as a method for distributing content, archiving data, and managing information. Given the portability and interchangeability of the recording media, optical technologies have found wide use in consumer and business applications. Propelling the development of optical recording has been the need in consumer electronics for higher data rates and increased storage capacity. For example, the progression of optical storage from capturing high-fidelity stereo audio to high-definition movies has required the evolution from compact discs to digital versatile discs to high-definition DVDs and Blu-ray discs (see Figure 1). ${ }^{1}$ In addition, the increasing performance requirements for archiving data have pushed the advancement of magneto-optical recording. ${ }^{2}$ Underlying the progress in optical storage has been continual advances in the recording materials to enable the higher storage capacities and faster data recording and readout rates.

The era of commercial optical data storage $^{3}$ began with the invention of "video disk" technology, later known as "laserdisc" technology, by David Paul Gregg in 1959. Designed for the distribution of video content, the approach used discs containing surface structures called pits and lands which modulate a readout light beam. The modulated light is captured by a pickup head and translated for playback. Initially configured using transparent discs and followed by more efficient reflective discs, laserdiscs were demon- strated to the public in 1972. The technology, which used 30-cm-diameter discs and stored video in analog format, was made commercially available in 1978. The laserdisc was an early demonstration of the power of optical storage: the absence of physical contact between the readout head and media, random access to content, and the relative simplicity of the media structure.

Building upon laserdisc technology, companies began developing optical discs capable of storing digital audio. In 1983, compact discs were introduced, storing approximately 74 minutes of audio. CDROM (read-only memory) discs, targeted for data storage and able to hold 650 Mbytes, entered the market soon after, followed by recordable and rewritable versions. Typically $1.2 \mathrm{~mm}$ thick and $120 \mathrm{~mm}$ in diameter, a CD-ROM consists of a single disc fabricated from polycarbonate. In the read-only structure, the polycarbonate is molded with pits $500 \mathrm{~nm}$ wide and $800 \mathrm{~nm}$ to $3.5 \mu \mathrm{m}$ long on its surface. A thin layer of metal is deposited on the surface of the disc. A semiconductor laser at $780 \mathrm{~nm}$ is used to reflect off of the pit and land structure of the disc to reconstruct the recorded data.

The early 1990s saw efforts to increase the storage capacity of these types of optical discs, resulting in the commercial introduction of digital video discs-or, as they have come to be called, digital versatile discs (DVDs) - in 1996. Unlike the single-disc construction of compact discs, DVDs consist of two 0.6-mm-thick polycarbonate discs coated with a thin metallic layer, with each layer containing data that can be read independently. The discs are bonded together to product a $1.2-\mathrm{mm}$ -

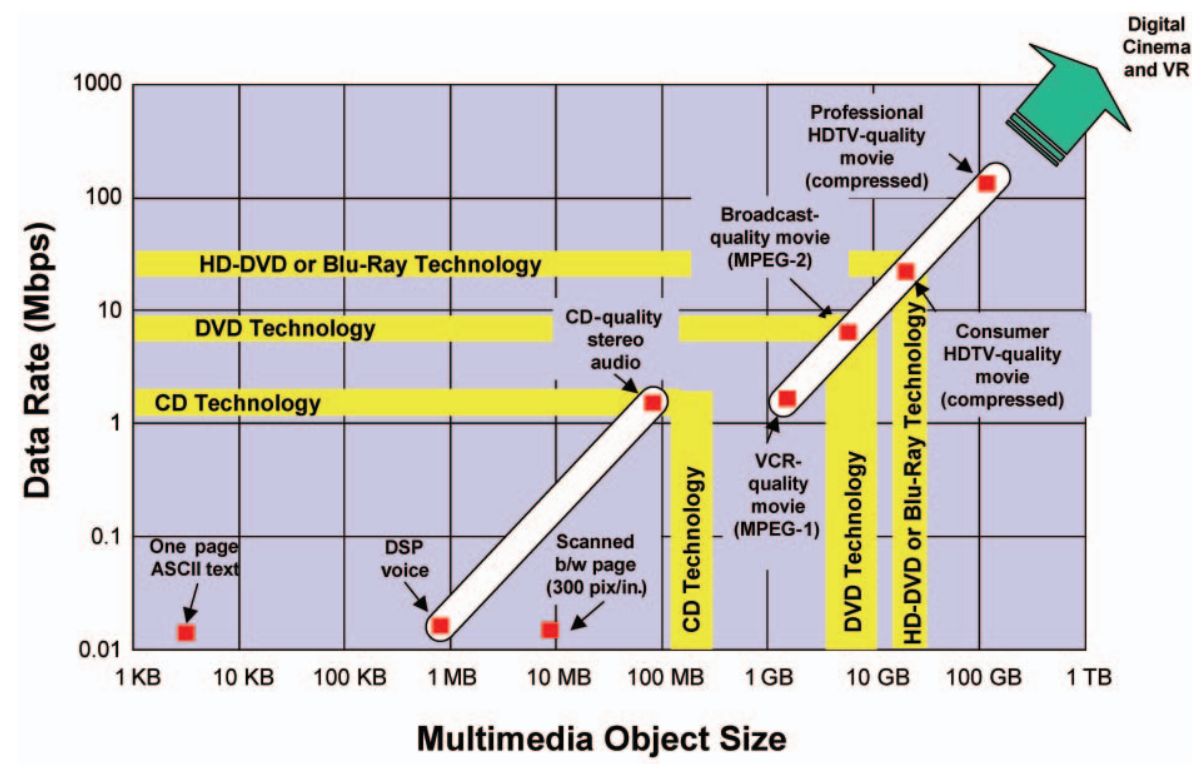

Figure 1. The continuing increase in storage capacity and complexity of multimedia objects has driven improvements in optical storage technologies. $C D=$ compact disc, $D S P=$ digital signal processor, $D V D=$ digital versatile disc, $G B=$ gigabytes, $H D-D V D=$ high-definition $D V D, K B=$ kilobytes, $M B=$ megabytes, pix = pixel, Mbps = megabits per second, MPEG-1 = Moving Pictures Expert Group Standard 1, MPEG-2 = Moving Pictures Expert Group Standard 2, TB = terabytes, VR = virtual reality. 
thick package. The discs can be read from one or both sides, with each side containing one or two layers of data. In addition to taking advantage of the capability of optics to focus on different depths in the media, the increase in storage capacity of DVDs over CDs is achieved by using smaller feature sizes and a shorter-wavelength readout laser $(650 \mathrm{~nm})$. A single-sided, single-layer DVD can hold 4.7 Gbytes of information. Efforts to increase the storage capacity have centered on the use of blue lasers with a shorter ( $405 \mathrm{~nm}$ ) wavelength, which allows smaller data bits and therefore higher capacity. These efforts are marked by the recent introduction of Bluray and high-definition DVDs that enable capacities of 15-30 Gbytes.

Concurrently with the development of CDs and DVDs, magneto-optical discs were developed, primarily for professional applications and the rewritable data market. Introduced in the late 1980s, this technology relies on the modulation of light by the magnetic state of the material. The performance of these discs has evolved from capacities of 650 Mbytes on a two-sided, 130-mm disc to current capacities of many gigabytes, with future products expected to reach terabytes of storage capacity.

In this issue of MRS Bulletin, we have sought to cover areas in materials research and engineering that span the field of optical storage. From the optimization of substrate materials for optical storage, to the growing complexity of materials for magneto-optic recording, to the recent developments of materials for blue laser recording, we hope to highlight the sophisticated design and fabrication methods that underpin the currently available optical storage technologies. In addition, we point to the future by including articles on materials for multilayer optical recording and holographic data storage, two technologies that can extend the optical storage roadmap to even higher performance. We conclude this issue with a contribution that summarizes the process and requirements for media standardization, the key for interchangeability in optical storage.

The importance of optimizing the materials properties of substrates for the various disc formats-CDs, DVDs, and blue laser discs-and issues such as media replication, recording, readout processes, and lifetime characteristics are discussed in the article by Bruder et al. in this issue. In particular, the authors focus on the development of polycarbonate materials that are not only tuned to exhibit the required performance profiles, but also, just as important, able to be cost-effectively manufactured.

The recent development of blue laser recording has extended the performance capabilities of the family of optical storage represented by CDs and DVDs. In their article, Kuiper and Pieterson write of the materials used in both write-once and rewriteable blue laser materials. The authors discuss developments in phasechange materials, spin-coated dye systems, and inorganic alloy materials and their suitability for the smaller optical spot sizes enabled by blue lasers.

We then examine the rare-earth/ transition-metal materials and structures central to magneto-optical recording in the article by Kaneko. The author traces the evolution of magneto-optical recording from its commercial introduction in 1988, through the emergence of super-resolution techniques that enabled resolution beyond the optical limit, to current discussions of extensions of super-resolution techniques.

In the article by Milster and Zhang, the authors examine the materials required for multilayer recording, a technique that aims to increase the capacity of optical disc recording by using multiple layers of bitwise-recorded data. The challenges presented by the interplay between materials properties and the optical systems used for recording are summarized.
The last article, on materials for optical storage, focuses on high-performance polymers for holographic data storage. Holographic storage represents a departure for optical technologies from the traditional bitwise approach to recording; data is stored throughout the volume of the recording media in a parallel, rather than serial, fashion. Dhar summarizes the materials requirements of media for holography and focuses on recent progress in the development of polymer recording materials.

Glinka ends the issue with an article on the processes required for optical recording media and the organizations involved in standardizing those processes. For the established optical storage technologies, this standardization has been the key to their broad adoption. As emerging technologies such as multilayer recording and holographic storage reach commercialization and then widespread use, standardization will become a part of their development as well.

We hope that these articles focused on materials for established optical storage technologies will draw attention to the technological challenges involved in optimizing commercially viable materials. The strategies used to optimize materials with respect to issues such as performance, environmental robustness, and manufacturability have been critical in extending the capabilities of established optical technologies. These strategies serve as models for the development of materials for the emerging areas of blue, multilayer, and holographic recording.

\section{References}

1. J. Taylor, DVD Demystified (McGraw-Hill, New York, 2000).

2. R.J. Gambino and T. Suzuki, eds., MagnetoOptical Recording Materials (IEEE Press, Piscataway, NJ, 2000).

3. K. Immink, J. Audio Eng. Soc. (1998) p. 458.

\begin{abstract}
Hans Coufal, guest editor for this issue of MRS Bulletin, is manager of device and systems innovation in the IBM Research Center. He is currently on assignment to the Semiconductor Research Corp. as the founding director of the Nanoelectronics Research Corp.

Coufal earned his applied physics degrees
\end{abstract}

from the Technische Hochschule in Munich, Germany. After serving as a junior faculty member at the same university and at the Freie Universität in Berlin, he joined the staff at the IBM San Jose Research Laboratory, the precursor to the Almaden Research Center, in 1981. He has held several management positions in addition to his own scientific work on thermal and acoustic transients and holographic data storage. He has more than 150 scientific publications, is the editor of eight books, and holds 14 patents. He is a fellow of the Optical Society of America, the American Physical Society of America, and the International Union

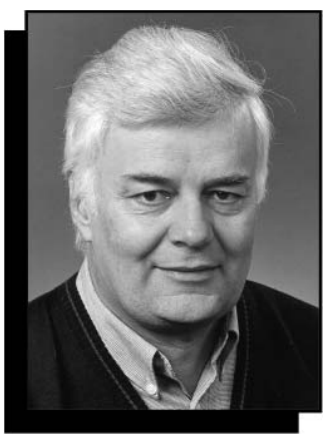

Hans Coufal

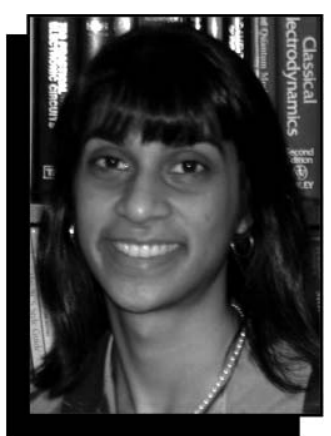

Lisa Dhar 
of Pure and Applied Physics. He is also the recipient of the Bundes-verdienstkreuz, the German counterpart of the Order of Merit.

Coufal can be reached at IBM Almaden Research Center, KLPA/D2, IBM Research Division, 650 Harry Road, San Jose, CA 95120-6099, USA; tel. 408-927-2441 and e-mail coufal@almaden. ibm.com.

Lisa Dhar, guest editor for this issue of $M R S$ Bulletin, is the vice president of media development and one of the founders of InPhase Technologies (Longmont, Colo.). Before founding InPhase, she was a member of the Bell Labs core team at Lucent Technologies responsible for developing the technology in holographic storage upon which InPhase is based. She holds a PhD degree from the Massachusetts Institute of Technology and a BS degree from the University of Chicago, both in chemistry. She has authored numerous technical articles in the areas of holographic data storage, optical microscopy, and pulsed lasers, and she is the inventor on more than 15 patents and patent applications.

Dhar can be reached at InPhase Technologies, 2000 Pike Road, Longmont, CO 80501, USA; tel. 217-369-7409 and e-mail lisadhar@ inphase-tech.com.

Friedrich-Karl Bruder is a senior scientist in the polymer physics department within the polycarbonates business unit of Bayer MaterialScience AG in Krefeld, Germany.

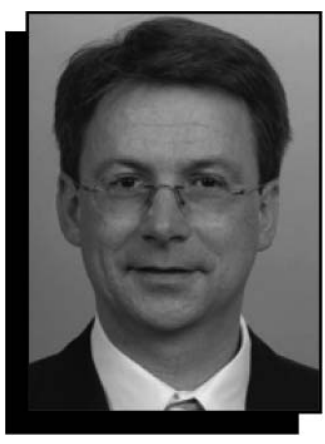

Friedrich-Karl Bruder

He studied physics at the University of Freiburg, where he obtained his PhD degree in 1992 investigating the mixing and de-mixing behavior of polymer blends near surfaces and interfaces. He then joined Bayer's central research department as head of one of the physics laboratories. Since then, Bruder has worked on various research projects related to structure-property relations of polycarbonates, electroconductive polymers, DVD-grade polycarbonates, copolycarbonates with low birefringence, and hydrogenated styrene polymers and dye development for recordable optical discs. His current projects are mainly in the field of optical data storage, such as the improvement of polycarbonate for future formats such as Blu-ray discs, highdensity DVDs, substrate materials, and functional materials for nearfield recording and holographic data storage.

Bruder can be reached at Bayer MaterialScience AG, BU Polycarbonates, Global Innovations \& Product Management, Polymer Physics, Bldg. R79, 47812 Krefeld,

Germany; e-mail friedrich-karl.bruder@ bayerbms.com.

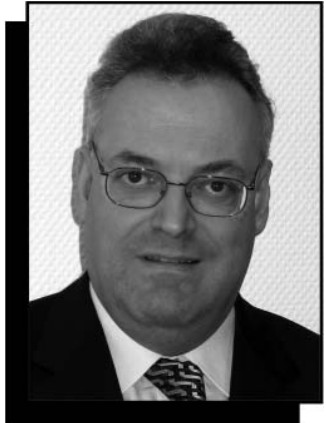

Konstantinos Douzinas

Konstantinos Douzinas is head of the polymer physics department within the polycarbonates business unit of Bayer MaterialScience AG in Krefeld, Germany, a position he has held since 2004. After obtaining his diploma in chemical engineering from the National Technical University in Athens, Greece, he received his $\mathrm{PhD}$ degree in chemical engineering from MIT in 1991. The same year, he joined Bayer AG Central Research in Leverkusen, Germany, and worked on new materials for industrial, medical, and data storage applications. In 2001, he joined the physics department of Bayer AG and took over responsibility for a working group dealing with optical storage systems.

Douzinas can be reached at Bayer MaterialScience AG, BU Polycarbonates, Global Innovations \& Product Management, Polymer Physics, Bldg. R79, 47812 Krefeld, Germany; e-mail konstantinos. douzinas@bayerbms.com.

Uli Franz is the global industry manager responsible for the worldwide coordination of optical data storage activities in the polycarbonates business unit of Bayer MaterialScience

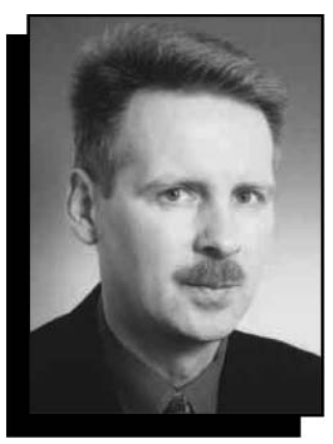

Uli Franz

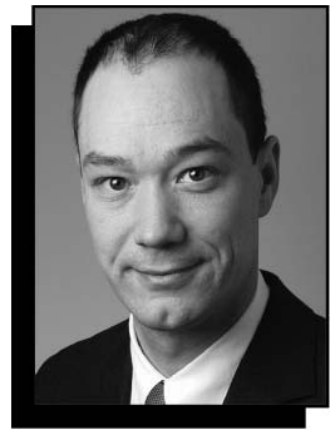

Rafael Oser

AG in Leverkusen, Germany. He received his $\mathrm{PhD}$ degree in macromolecular chemistry at the Technische Universität in Munich. In 1994, he joined Miles (now Bayer Corp.) in Pittsburgh, Pa., and worked on different projects in the corporate polymer research group. In 1996, he joined the plastics division and was responsible for the technical marketing of polycarbonate in various application areas. In 2000, he transferred to Bayer AG in Leverkusen, taking over responsibility as global industry manager for optical data storage applications.

Franz can be reached at Bayer AG, 51368 Leverkusen, Germany; e-mail uli.franz@ bayerbms.com.

Werner Glinka founded the Glinka Company in Woodside, Calif., in 1999 and is the chairman of Ecma TC44, an interna-

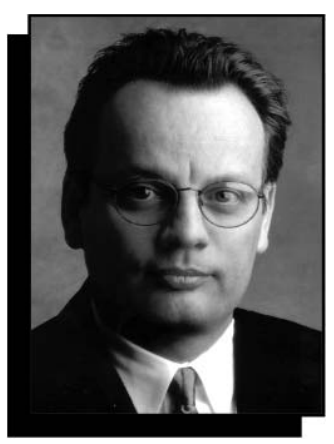

Werner Glinka

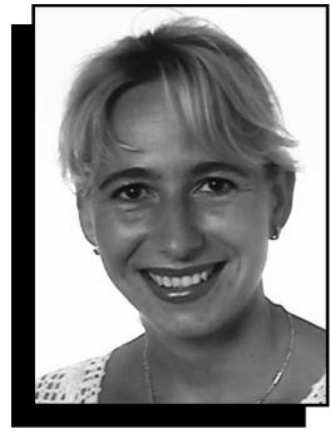

Liesbeth van Pieterson

tional standards committee for holographic storage systems. He received his degree in electrical engineering from Fachhochschule Bochum in Germany and has more than 25 years of experience in product marketing, design engineering, and industry standards. $\mathrm{He}$ was responsible for marketing and product planning for optical storage and system products at Sony America, served as senior director of marketing of optical storage and multimedia products for Hitachi America Ltd., and has also held senior marketing and engineering positions at Maxoptix/Maxtor Corp.,

Laserdrive Ltd., Memo-

rex, and Nixdorf

Computers.

Glinka was chair of the Fibre Channel Industry Association (FCIA) from 2004 to 2005 and founding executive director of the 


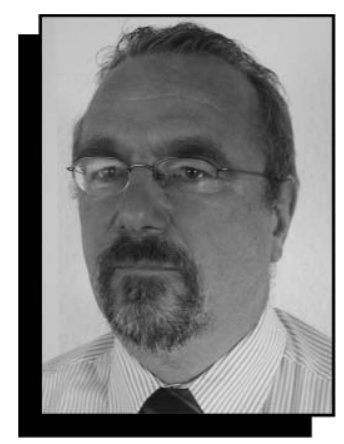

Wilfried Haese

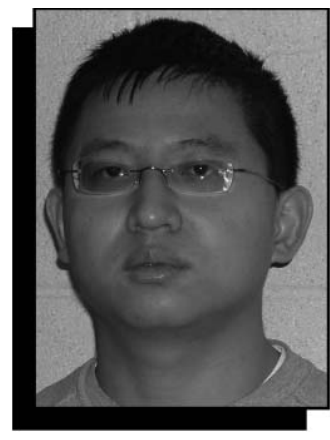

Yan Zhang

DAFS Collaborative (2002). He can be reached at the Glinka Company, 214 Swett Road, Woodside, CA 94062, USA; tel. 650-8515909, fax 650-851-5987, and e-mail werner. glinka@theglinkacompany. com.

Wilfried Haese is a senior scientist in the polycarbonate innovations department of Bayer MaterialScience AG in Krefeld, Germany. He received his $\mathrm{PhD}$ degree in organic chemistry at the Technische Hochschule Aachen. In 1983, he joined Bayer AG in Leverkusen and worked on a variety of projects in central research. In 1989, he joined the plastics division and was responsible for developing application technologies for liquid-crystalline polyesters and poly(phenylene sulfide). In 1992, he joined the polycarbonate group

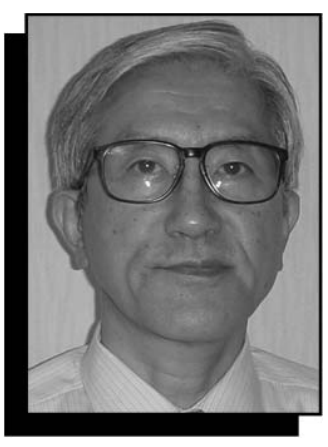

Masahiko Kaneko

and has specialized in optical data storage applications since 1995. Currently, he is responsible for the development of polycarbonate substrate materials for optical data storage media.

Haese can be reached at Bayer MaterialScience AG, BU Polycarbonates, Global Innovations \& Product Management, Polymer Physics, Bldg. R79, 47812 Krefeld, Germany; e-mail wilfried. haese@bayerbms.com.

Masahiko Kaneko has been with Sony Corporation since 1976 and is currently a principal researcher and deputy leader of a national project for the development of ultrathin, smalldiameter disc drives. $\mathrm{He}$ received his BS degree from the Tokyo Institute of Technology in 1970 and his MS and PhD degrees in optical studies of magnetic materials from Tokyo University in 1972 and 1975, respectively.

Kaneko began his work at Sony researching magnetic powders and LPE (liquid-phase epitaxy) garnet films. He was then engaged in the development of magneto-optical materials, especially magnetic multilayers for direct overwriting, magnetic super-resolution, and DWDD (domain-wall

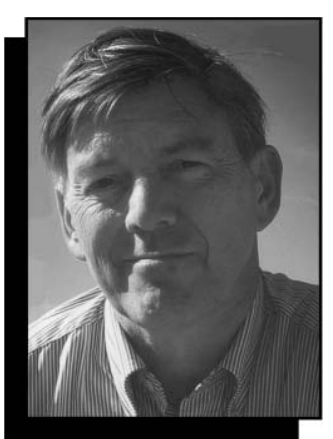

A.E.T. (Ton) Kuiper

displacement detection) media. Before beginning his current project, he was a national project group leader for the development of a 100Gbyte multilevel read-only memory disc.

Currently, he is also a visiting professor at the Tokyo Institute of Technology and is the general chair of the MORIS (Magneto-Optical Recording International Symposium) 2006 Workshop on Thermal and Optical Magnetic Materials and Devices, which will be held in June. He is a member of the Magnetic Society of Japan, the Physical Society of Japan, and the Institute of Electrical Engineers of Japan.

Kaneko can be reached at Sony Corporation, Optical System Development Division, Video Business Group, Home Electronics Network Company, 6-7-35 Kitashinagawa, Shinagawaku, Tokyo, 141-0001 Japan; tel. 81-35795-6193, fax 81-3-57956230, and e-mail Masahiko.Kaneko@jp. sony.com.

A.E.T. (Ton) Kuiper is a principal scientist at Philips Research Laboratories in Eindhoven, the Netherlands. He received his $\mathrm{PhD}$ degree in catalysis at the University of Technology in Eindhoven in 1974 and

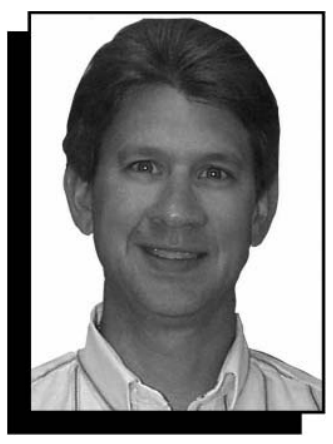

Tom D. Milster

joined Philips Research in 1975. His past work has been related to thin films, ranging from surface science, deposition, and analysis to thin-film magnetic heads and sensors. His current activities are in optical storage media, with an emphasis on phasechange materials. He is a member of the Dutch Vacuum Society and the editorial board of Thin Solid Films.

Kuiper can be reached at Philips Research Eindhoven, WAG-12, HTC 04, 5656 AE Eindhoven, Netherlands; tel. 31-40-2747984, fax 31-40-2746321, and e-mail ton.kuiper@ philips.com.

Tom D. Milster is a research professor of optical sciences at the University of Arizona. He received his BS degree in electrical engineering from the University of Missouri in 1981 and his PhD degree from the University of Arizona's Optical Sciences Center in 1987. He worked as an optical engineer in IBM's general products division before joining the faculty of the University of Arizona as an assistant professor.

His major research interest is the study of physical phenomena in high-performance optical systems. In particular, his research involves microscopy, lithography, and optical data storage.

Milster is a fellow of the Optical Society of America and the International Society of Optical Engineering (SPIE).

His research activities have led to more than 100 papers as well as numerous contributions and invited presentations at national and international conferences. He also designed an extreme-ultraviolet spectrometer which was part of the scientific package that flew in the space shuttle with Sen. John Glenn in 1998.

Milster can be reached at the University of Arizona, Optical Sciences Center, 1630 East University Blvd., Tucson, AZ 85721, USA; tel. 520-621-8280, fax 520-621-4358, and e-mail milster@arizona.edu.

Rafael Oser is a research scientist in the polymer physics department of the polycarbonates business unit of Bayer MaterialScience AG in Krefeld, Germany. He received his diploma in physics from the University of Karlsruhe in 1995. After completing his doctorate at the University of Freiburg in polymer physics, he joined Bayer AG Central Research in 2000. His interests focus on optical data storage materials and thin-film technologies.

Oser can be reached at Bayer MaterialScience AG, BU Polycarbonates, Global Innovations \& Product Management, Polymer Physics, Geb. R79, Raum 452, 47812 Krefeld, Germany; e-mail rafael.oser@ bayermaterialscience.com.

Liesbeth van Pieterson is a senior scientist at Philips Research Labo- 
ratories in the Netherlands. She received her $\mathrm{PhD}$ degree in chemistry of condensed matter from Utrecht University in 2001 and began her work at Philips Research that same year. Her research focus is in the area of solid-state chemistry with an emphasis on the physics and chemistry of materials for optical recording.

In 2002, she received the PUG award, a prize given every four years for the best thesis in beta sciences at Utrecht University. She is a member of the program committee of the European Symposium on
Phase-Change and Ovonic Science (EPCOS), and she is treasurer of the Dutch Vacuum Society (NEVAC).

Liesbeth van Pieterson can be reached at Philips Research, WAG12, HTC 04, 5656 AE Eindhoven, Netherlands; tel. 31-40-27-42343, fax
31-40-27-46505, and e-mail liesbeth.van. pieterson@philips.com.

Yan Zhang is a postdoctoral fellow in the School of Optometry at Indiana University. He graduated from the University of Arizona's Optical Sciences Center in 2004. His research interest centers on high-resolution threedimensional imaging.

Zhang can be reached at Indiana University, School of Optometry, 800 E. Atwater Ave., Bloomington, IN 47405, USA; e-mail yz7@ indiana.edu.

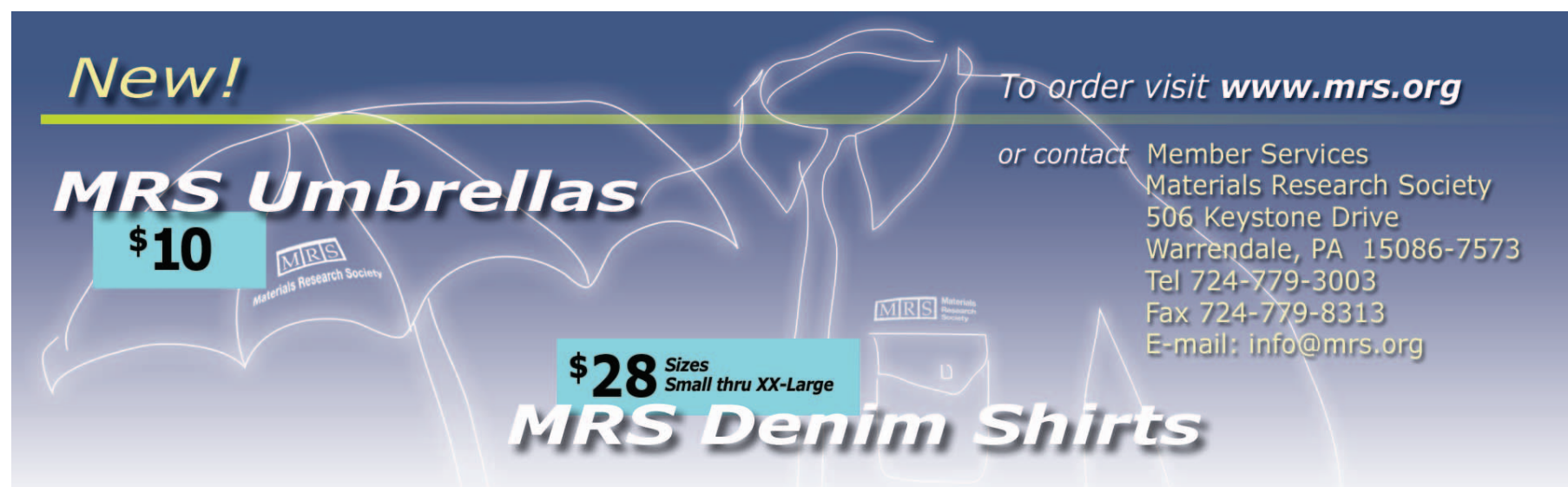

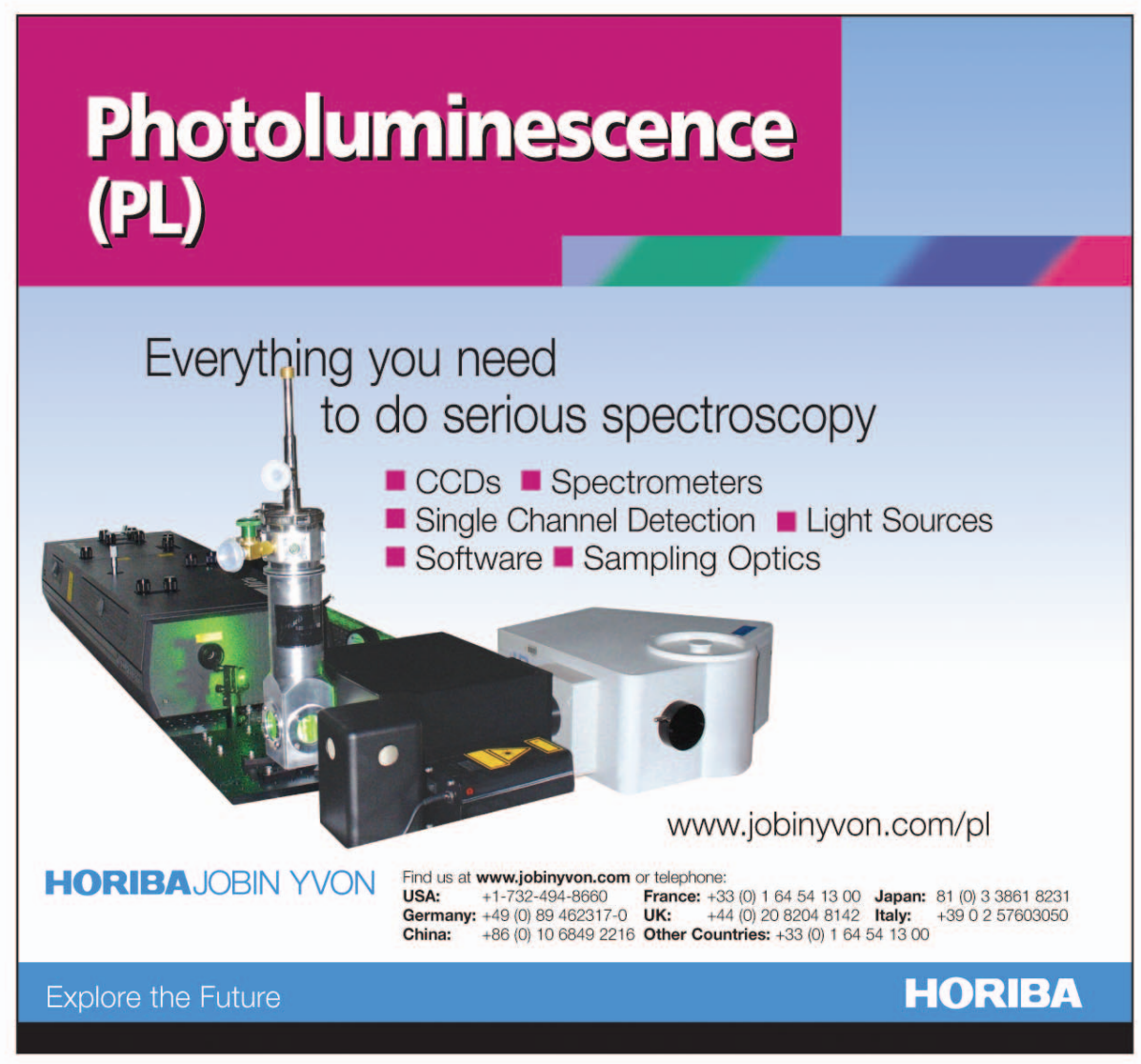

For more information, see http://www.mrs.org/bulletin_ads

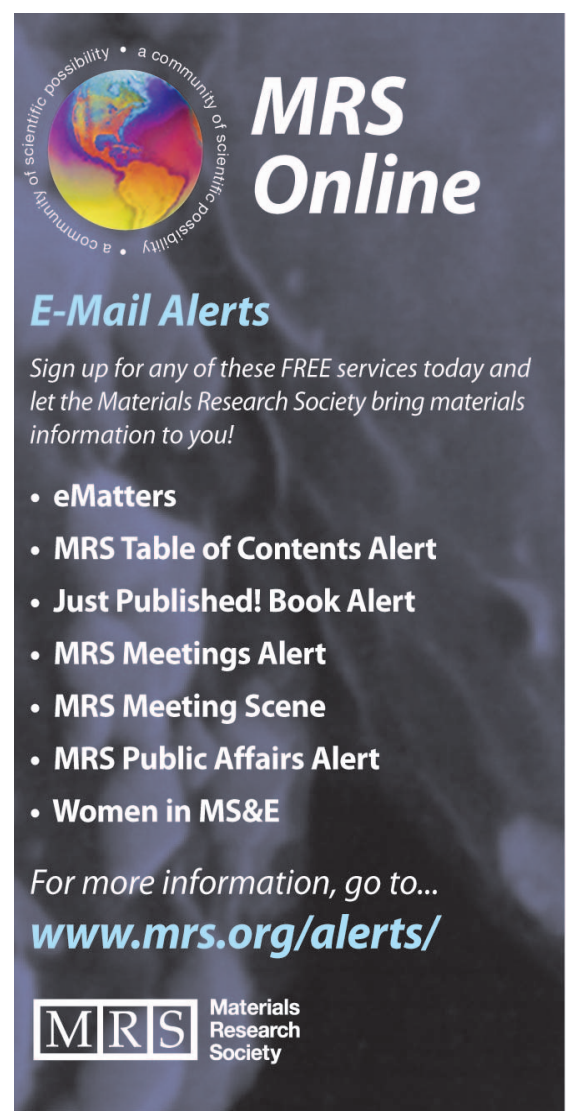

MRS BULLETIN • VOLUME 31 • APRIL 2006 